BNL-108347-2015-JA

\title{
Decreasing Transmembrane Segment Length Greatly Decreases Perfringolysin O Pore Size
}

\author{
Qingqing Lin ${ }^{1}$ Tong Wang $^{2}{ }^{\text {Huilin }} \mathrm{Li}^{1,2}{ }^{\bullet}$ Erwin London ${ }^{1}$ \\ ${ }^{1}$ Department of Biochemistry and Cell Biology, Stony Brook University, \\ Stony Brook, NY 11794-5215, USA \\ 2 Biosciences Department, Brookhaven National Laboratory, \\ Upton, NY 11973, USA
}

Submitted to Journal of Membrane Biology

January 2015

\section{Biological, Environmental \& Climate Sciences Department Brookhaven National Laboratory}

\section{Prepared for U.S. Department of Energy}

\footnotetext{
Notice: This manuscript has been authored by employees of Brookhaven Science Associates, LLC under Contract No. DE-SC0012704 with the U.S. Department of Energy. The publisher by accepting the manuscript for publication acknowledges that the United States Government retains a non-exclusive, paid-up, irrevocable, world-wide license to publish or reproduce the published form of this manuscript, or allow others to do so, for United States Government purposes.
} 


\section{DISCLAIMER}

This report was prepared as an account of work sponsored by an agency of the United States Government. Neither the United States Government nor any agency thereof, nor any of their employees, nor any of their contractors, subcontractors, or their employees, makes any warranty, express or implied, or assumes any legal liability or responsibility for the accuracy, completeness, or any third party's use or the results of such use of any information, apparatus, product, or process disclosed, or represents that its use would not infringe privately owned rights. Reference herein to any specific commercial product, process, or service by trade name, trademark, manufacturer, or otherwise, does not necessarily constitute or imply its endorsement, recommendation, or favoring by the United States Government or any agency thereof or its contractors or subcontractors. The views and opinions of authors expressed herein do not necessarily state or reflect those of the United States Government or any agency thereof. 


\title{
Decreasing Transmembrane Segment Length Greatly Decreases Perfringolysin O Pore Size
}

\author{
Qingqing Lin ${ }^{1}$, Tong Wang ${ }^{2}$, Huilin $L^{1,2}$ and Erwin London ${ }^{1, *}$ \\ ${ }^{1}$ Department of Biochemistry and Cell Biology, Stony Brook University, Stony Brook, NY 11794-
} 5215

${ }^{2}$ Biosciences Department, Brookhaven National Laboratory, Upton, NY 11973

*To whom correspondence should be addressed: Department of Biochemistry and Cell Biology, Stony Brook University, Stony Brook, NY 11794-5215.

Tel: 631-632-8564

Email: Erwin.London@stonybrook.edu

Running Title: PFO Transmembrane Pore Size

This work was supported by NSF grant MCB 1019986. 


\begin{abstract}
Perfringolysin $O$ (PFO) is a transmembrane (TM) $\beta$-barrel protein that inserts into mammalian cell membranes. Once membrane-inserted, PFO assembles into pore-forming oligomers containing 30-50 PFO monomers. These form a pore of up to $300 \AA$, far exceeding the size of most other proteinaceous pores. In this study, we found that altering PFO TM segment length can alter the size of PFO pores. A PFO mutant with lengthened TM segments oligomerized to a similar extent as wild type PFO, and exhibited pore-forming activity and a pore size very similar to wild type PFO as measured by electron microscopy and a leakage assay. In contrast, PFO with shortened TM segments exhibited a large reduction in pore-forming activity, and pore size. This suggests that the interaction between TM segments can greatly affect the size of pores formed by TM $\beta$-barrel proteins. PFO may be a promising candidate for engineering pore size for various applications.
\end{abstract}


Perfringolysin O (PFO) belongs to the family of cholesterol-dependent cytolysins (CDCs). CDCs contribute to the pathogenesis of a large number of Gram-positive bacterial pathogens (Parker et al. 2005; Aroian et al. 2007). These toxins bind to cholesterol in the membranes of eukaryotic cells and oligomerize into ring-shaped structures, estimated to contain 30-50 subunits, which surround pores of 30-35 nm diameter (Tweten 2005; Hotze et al. 2012). This size by far exceeds that of most other large proteinaceous pores, e.g. staphylococcal $\alpha$-toxin with a central pit of 2-3 nm in diameter (Fussle et al. 1981) or even the complement membrane attack complex with an internal pore up to $10 \mathrm{~nm}$ (Bhakdi et al. 1984). PFO pores permit flux of both ions and macromolecules, and the toxin has become a popular tool for the controlled permeabilization of cell membranes (Cassidy et al. 2013).

Crystallographic studies on PFO show that the monomeric toxin molecule is elongated and contains four domains (named domains 1-4) rich in $\beta$-sheet structure (Rossjohn et al. 1997). Upon binding to a target membrane, bound PFO molecules diffuse laterally and interact with each other to form a prepore complex (Shepard et al. 2000). Subsequently, a profound conformational change occurs that results in the formation of the transmembrane (TM) pore. Using a combination of cysteine scanning and fluorescence spectroscopy, Shepard et al. (Shepard et al. 1998) and Shatursky et al. (Shatursky et al. 1999) identified the membranespanning region of PFO. Two sets of $\alpha$-helical bundles that flank the domain 3 core $\beta$-sheets, which extend from the domain 3 core $\beta$-strands 1-4, undergo a change in their secondary structure to form two amphipathic $\beta$-hairpins. Shatursky et al. (Shatursky, Heuck et al. 1999) further showed that these twin hairpins spanned the bilayer. Each TM hairpin (which has been designed by the term TMH (Shatursky, Heuck et al. 1999)) is approximately 30 residues long, and contains two $\beta$-strands. In a previous study we showed that altering the length of PFO TM segments affects PFO localization into liquid ordered or liquid disordered membrane microdomains (Lin et al. 2013). PFO mutants with lengthened or shortened TM segments also exhibited different pore formation activity, which was dependent on membrane thickness. Here, we report that altering the length of PFO TM segments can unexpectedly alters pore and oligomer size. This is of interest because it has implications for bioengineering of proteins with specific pore properties. Lengthening the strand length by two residues did not greatly affect the pore or oligomer size, but did decrease the stability of oligomerization. Shortening strands by two residues had a larger effect, greatly reducing the pore and oligomer size. These findings suggest engineering PFO TM strand length may be a promising approach to constructing PFO molecules that can allow the cellular escape or delivery of macromolecules with a highly controlled size limit.

\section{MATERIALS AND METHODS \\ Materials}

Phospholipids: 1,2-dimyristoleoyl-sn-glycero-3-phosphocholine (DMoPC), 1,2-dioleoyl-snglycero-3-phosphocholine (DOPC), 1,2-distearoyl-sn-glycero-3-phosphocholine (DSPC), cholesterol (ovine wool), 1,2-[9,10-dibromo] stearoyl-sn-glycero-3-phosphocholine (BrPC), and 1,2-dioleoyl-sn-glycero-3-phosphoethanolamine-N-(lissamine rhodamine B sulfonyl) (RhoDOPE) were purchased from Avanti Polar Lipids (Alabaster, AL). Lipids were stored in ethanol or chloroform at $-20^{\circ} \mathrm{C}$. Concentrations were determined by dry weight or by absorbance, using an $\varepsilon$ of $95,000 \mathrm{~cm}^{-1} \mathrm{M}^{-1}$ at $560 \mathrm{~nm}$ for rhodamine lipids in methanol (Nelson et al. 2010). 
Streptavidin was purchased from GenScript (Piscataway, NJ). 4,4-difluoro-5,7-dimethyl-4-bora3a,4a-diaza-s-indacene-3-propionic acid (BODIPY-FL), cascade blue (CB) labeled dextran (CBdextran) (molecular weight $3 \mathrm{kD}, 10 \mathrm{kD}, 70 \mathrm{kD}$ ) and the free cascade blue dye, 8methoxypyrene-1,3,6-trisulfonic acid, trisodium salt (MPTS) were purchased from Molecular Probes, Invitrogen (Grand Island, NY). Biocytin ( $\varepsilon$-biotinoyl-L-lysine) was purchased from Anaspec (San Jose, CA). All other chemicals were reagent grade. BODIPY-labeled streptavidin (BOD-SA) was prepared by following protocols developed by A. Heuck (U. Mass. Amherst). Generally, streptavidin and BODIPY-FL were mixed at 4:1 mole ratio in $0.1 \mathrm{M} \mathrm{NaHCO}_{3}, \mathrm{pH} 8.3$ and incubated for $2 \mathrm{~h}$ at room temperature. The reaction mixture was then applied to Sephadex G-50 to separate the conjugate from unreacted BODIPY-FL. The concentration of BOD-SA was determined by absorbance, using an $\varepsilon$ of $41,326 \mathrm{~cm}^{-1} \mathrm{M}^{-1}$ at $280 \mathrm{~nm}$.

\section{Generation and purification of PFO derivatives}

A functional cysteine-less derivative of wild type PFO (PFO C459A) (Shepard, Heuck et al. 1998) were a kind gift of A. Heuck, U. Mass. Amherst. PFO mutants with lengthened (long PFO) or shortened (short PFO) TM sequences were generated as previously described from the PFO C459A plasmids using site-directed ligase-independent mutagenesis (Chiu et al. 2004; Chiu et al. 2008). Long PFO contains 2 Ala inserted between residues V202-L203; E204-N205; I298-K299, and N300-T301 respectively, and short PFO has residues K201V202, N205S206, L297I298 and T301D302 deleted (Lin and London 2013). Variants of PFO with an Ala to Cys substitution at residue 215 were generated by the QuickChange site-directed mutagenesis kit (Stratagene) as previously described (Lin and London 2013). PFO was expressed in E. coli BL21(DE3)pLysS and purified similarly as described previously (Nelson et al. 2008). Final purity for WT and long PFO appeared to be $>95 \%$, and roughly $\sim 70-80 \%$ for short PFO, which only expresses at low levels. The purified PFO was stored in phosphate-buffered saline (PBS, $1.8 \mathrm{mM} \mathrm{KH_{2 } P O 4 , 1 0 \mathrm { mM }}$ $\mathrm{Na}_{2} \mathrm{HPO} 4,137 \mathrm{mM} \mathrm{NaCl}$, and $2.7 \mathrm{mM} \mathrm{KCl}$ at $\mathrm{pH} 7.4$ ) at a concentration of $\sim 1-1.5 \mathrm{mg} / \mathrm{ml}$ for WT and long PFO, or $\sim 0.3-0.5 \mathrm{mg} / \mathrm{ml}$ for short PFO at $-20{ }^{\circ} \mathrm{C}$.

\section{Preparation of lipid vesicles}

Multilamellar vesicles (MLV) were prepared with desired mixture of lipids. Lipids in solvent were mixed, and then dried with $\mathrm{N}_{2}$. They were then re-dissolved in $\mathrm{CHCl}_{3}$ and re-dried under $\mathrm{N}_{2}$ and then in high vacuum for at least $1 \mathrm{~h}$. The re-dried lipid mixtures were dispersed in PBS, pH 5.1 at $70{ }^{\circ} \mathrm{C}$ to give the desired final concentration and agitated at $55^{\circ} \mathrm{C}$ for 15 min using a VWR multitube vortexer (Westchester, PA) placed within a convection oven (GCA Corp, Precision Scientific, Chicago, IL). Samples were then cooled to room temperature.

For EM measurements, large unilamellar vesicles (LUV) were prepared from MLV by subjecting the MLV to seven cycles of freezing in a mixture of dry ice and acetone and thawing at room temperature. They were then extruded through 200-nm polycarbonate filters (Avanti Polar Lipids, Alabaster, AL) 11 times to obtain LUV of uniform vesicle size. LUV samples for leakage studies were prepared as described below.

\section{Fluorescence intensity measurements}

Fluorescence emission intensity was measured (unless otherwise noted) at room temperature on a SPEX Fluorolog 3 spectrofluorimeter. For fixed wavelength measurements, 
excitation and emission wavelength sets used (in $\mathrm{nm}$ ) were $(280,340)$ for tryptophan; $(400,420)$ for CB-dextran and MPTS; $(488,515)$ for BODIPY-FL labeled streptavidin (BOD-SA) and $(570$, 590) for Rho-DOPE. Unless otherwise noted, fluorescence intensity in single background samples lacking fluorophores was subtracted.

\section{Assay for biocytin leakage}

PFO-induced pore formation was measured by assaying the reaction of vesicle-trapped biocytin with externally added BOD-SA via the increase in the BODIPY fluorescence emission intensity upon binding of biocytin to BOD-SA located in the external solution, as previously described (Nelson, Johnson et al. 2008). Generally, $537 \mu \mathrm{M}$ biocytin was trapped within LUVs composed of $10 \mathrm{mM} \mathrm{6:4} \mathrm{(mol:mol)} \mathrm{DOPC/cholesterol.} \mathrm{After} \mathrm{dialysis} \mathrm{against} 4 \mathrm{~L}$ PBS buffer overnight, $10 \mu \mathrm{l}$ of vesicles were diluted with $990 \mu \mathrm{l}$ PBS pH 5.1. Then $10 \mathrm{nM}$ BOD-SA was added externally. BODIPY emission intensity was measured. Various amounts of WT, long, or short PFO were added and after a brief mixing, BODIPY intensity was monitored as a function of time for up to $40 \mathrm{~min}$.

\section{Entrapping BOD-SA, CB-dextran or MPTS in model membrane vesicles}

LUV containing trapped BOD-SA, trapped CB-dextran, or trapped MTPS were prepared similarly to as described previously (Rosconi et al. 2004; Wang et al. 2009). A mixture containing $10 \mathrm{mM}$ lipids comprised of 45:15:40 (mol:mol:mol) DOPC/BrPC/cholesterol or 27.5:27.5:45 (mol:mol:mol) DSPC/DMoPC/cholesterol with 0.002 mol\% Rho-DOPE (a fluorescent lipid marker), $70 \mu \mathrm{g} / \mathrm{ml}$ BOD-SA or $10 \mu \mathrm{M}$ CD-dextran or $10 \mu \mathrm{M}$ MPTS, and $20 \mathrm{mg} / \mathrm{ml} \mathrm{n}$-octyl- $\beta$ glucoside, was dissolved in $1 \mathrm{ml} \mathrm{PBS,} \mathrm{pH}$ 7.4. After removing $n$-octyl- $\beta$-glucoside by dialysis against 4 L PBS buffer at $4{ }^{\circ} \mathrm{C}$ overnight, the samples were extruded through 200-nm polycarbonate filters 11 times. Then a Sepharose 4B-CL column $(1 \mathrm{~cm}$ in diameter, $25 \mathrm{~cm}$ in length) was used to separate free BOD-SA, CB-dextran or MPTS from LUV-trapped BOD-SA, CBdextran or MPTS, respectively. After elution with PBS $\mathrm{pH} 7.4$, fractions ( $1 \mathrm{ml}$ per fraction) containing LUV (usually fractions 6-8) were collected for experiments. Typically the final lipid concentration was 2-4 mM as determined by Rho-DOPE fluorescence, i.e. by determining the ratio of the fluorescence of Rho-DOPE after sample preparation to that in the vesicles prior to fractionation, assuming that the loss in lipids during dialysis is minimal. Final average vesicle diameter as estimated by dynamic light scattering (Cheng et al. 2011) was $130 \mathrm{~nm}$.

\section{Assay for leakage of BOD-SA, CB-dextran or MPTS}

The leakage of BOD-SA, CB-dextran or MPTS was measured by diluting a 125-250 $\mu$ l aliquot of LUVs containing trapped BOD-SA, CB-dextran or MPTS to $1 \mathrm{ml}$ with PBS pH 5.1 (or pH 7.4 where noted) to give a final lipid concentration $\sim 500 \mu \mathrm{M}$ and final $\mathrm{pH}$ 5.3-5.7. Then the LUVs were incubated with small aliquots containing $20 \mu \mathrm{g}$ WT PFO, $20 \mu \mathrm{g}$ long PFO, or $30 \mu \mathrm{g}$ short PFO for $1 \mathrm{~h}$ at room temperature. Samples were then spun for $30 \mathrm{~min}$ in a Beckman L8-85 ultracentrifuge at $84,000 \mathrm{~g}$ at $4{ }^{\circ} \mathrm{C}$. After centrifugation, supernatants containing leaked BOD-SA or CB-dextran or MPTS were removed, and pellets containing the LUVs were resuspended in $1 \mathrm{ml}$ PBS pH 5.1. Rho-DOPE and either BOD-SA or CB fluorescence was then measured for both the supernatant and the pellet. (Calculated values have been corrected for incomplete pelleting of vesicles (see below).) 


\section{Electron microscopy and image processing}

$50 \mu \mathrm{g}$ WT, long, or short PFO was incubated with $1 \mathrm{ml}$ LUVs containing $500 \mu \mathrm{M}$ lipid, composed of 6:4 (mol:mol) DOPC/cholesterol or 27.5:27.5:45 (mol:mol:mol) DSPC/DMoPC/cholesterol in PBS pH 5.1 for at least $1 \mathrm{~h}$ at room temperature. To prepare EM grids for negative staining, a layer of carbon film ( $20 \mathrm{~nm}$ thick) was evaporated on a piece of freshly cleaved mica in an Edwards vacuum evaporator $\left(<10^{-5} \mathrm{Torr}\right)$. Carbon film was then peeled off from mica by floating it on the surface of deionized water and deposited on 300 mesh copper grids. All homemade continuous carbon coated grids were air-dried and stored for future usage. Carbon coated grids were first glow-discharged in 100 mTorr argon atmosphere for $1 \mathrm{~min}$. A drop of $4 \mu \mathrm{l}$ of sample solution was applied to the glow-discharged continuous carbon coated EM grid and incubated for $1 \mathrm{~min}$. Excess solution was removed by blotting the grid with a piece of filter paper. The grid was then washed by a drop of $\mathrm{dd}_{2} \mathrm{O}$ and stained in $2 \%(w / v)$ uranyl acetate aqueous solution for $1 \mathrm{~min}$. After blotting excess stain solution, the grid was air-dried. The negatively stained sample was examined in a JEOL JEM-2010F TEM operating at $200 \mathrm{kV}$. EM images were recorded in low-dose mode $\left(15 \mathrm{e}^{-} / \AA^{2}\right)$ at $50,000 \times$ or $60,000 \times$ microscope magnification using a Gatan UltraScan 4000 CCD camera (4,096 × 4,096 pixel).

Particle selection and image processing were done using EMAN and EMAN2 software package (Ludtke et al. 1999; Tang et al. 2007). Raw particle images were selected in a semiautomatic manner with e2boxer.py in EMAN2. The saved raw images were low-pass filtered to $2 \mathrm{~nm}$ and manually inspected to remove "bad" particles (low contrast, contacting other particles). Reference-free 2D classifications of image data set were done with refine2d.py in EMAN.

\section{RESULTS}

\section{Appearance of PFO with different length of TM segments in electron microscopy.}

We previously studied and characterized some of the properties of PFO with shortened and lengthened TM segments, and observed that a deletion of two residues from each of the sequences that form the TM $\beta$-strands resulted in a reduction of the extent of pore formation, while inserting two residues into them did not (Lin and London 2013). To obtain more information about the origin of this behavior, EM was used to examine whether changing the length of PFO TM segments altered the overall properties of membrane-inserted PFO. Electron micrographs of cholesterol-containing membranes showed that PFO oligomers appear as rings and partial rings called arcs (Mitsui et al. 1979; Shepard, Shatursky et al. 2000). When we prepared PFO under conditions giving complete cholesterol-dependent membrane insertion (Nelson, Johnson et al. 2008), these structures were also seen in our samples of WT PFO. Rings had an estimated diameter of 30-40 nm, consistent with $\sim 30-40$ subunits per pore (Fig. 1A). Very similar rings and arcs were observed with long PFO (Fig. 1B). In both cases, rings and arcs were often not membrane associated, suggesting that they had fallen off from the vesicles during EM sample preparation. It should be noted that despite the similarity between the appearance of WT and long PFO oligomers, WT PFO showed the characteristic resistance to 
disassembly on SDS gels (Shepard, Shatursky et al. 2000), while long PFO oligomers dissociated into monomers on SDS gels (Supplemental Fig. 1).

In contrast to WT PFO, Fig. 1C shows large ring-shaped or arc-shaped structures were absent from samples of short PFO mixed with lipid vesicles under conditions in which it recognizes membrane cholesterol and inserts maximally ( 65\%) (Lin and London 2013). Instead, small oligomeric structures were observed. (The observation that the short PFO frequently appears to be dissociated from vesicles may reflect an even more facile dissociation from membranes relative to WT PFO due to its short TM segments, or simply low visibility of short PFO when embedded in membranes.) Given the size of these structures, and counting "blobs" of mass as a monomer, the micrographs are suggestive of the formation of tetramers (image C2) or perhaps pentamers (image C1) by short PFO. This is also roughly consistent with the size of these oligomers relative to the size of the larger rings formed by WT in the micrographs. These small oligomers show what appears to be a small central hole, suggesting the possibility short PFO would form a much smaller pore than by WT or long PFO. While this is suggestive, a strong argument cannot be made based on the EM data, especially for short PFO. The short PFO oligomers are not membrane-associated, which could result in them forming a structure different from that when membrane bound, and there certainly could be other potential perturbations arising from preparing samples for electron microscopy. Furthermore, we cannot rule out the possibility that the oligomers seen with short PFO simply assembled in solution. Therefore, a different approach was used to investigate PFO pores (see below).

\section{Dose-dependent pore formation by WT, long and short PFO}

To investigate the suggestion from EM that short PFO has different pore properties than WT and long PFO, while the pore properties of WT and long PFO are similar, we first examined the extent of pore formation by measuring the efflux of the small molecule biocytin (biotinyllysine) out of vesicles containing membrane-inserted PFO. Upon efflux of biocytin from vesicles, it binds to the externally-added BODIPY-streptavidin (BOD-SA). This displaces the BODIPY group covalently bound to streptavidin from the biotin binding pocket on the streptavidin molecule, which results in an increase in BODIPY fluorescence (Nelson, Johnson et al. 2008). As shown in Fig. 2, under conditions in which WT and long PFO have been previously shown to be fully membrane-bound, and short PFO maximally membrane-bound (Lin and London 2013), WT and mutant PFO formed pores in DOPC vesicles containing 40 mol\% cholesterol. The extent of pore formation was protein dose-dependent. This is likely to reflect an increasing fraction of vesicles containing pores as the concentration of protein is increased (see below). With increasing amounts of WT PFO added, BODIPY emission intensity plateaued at $5 \mu \mathrm{g}$ WT PFO with a near three-fold increase in fluorescence. Similarly, after adding $50 \mu$ of 1:4 Triton X$100 /$ water to the samples to dissolve the vesicles and release trapped biocytin, the BODIPY intensity increase was 3-3.5 fold (data not shown), suggesting the plateau reflects the point when all of the BOD-SA is bound to biocytin.

Dose-dependent pore formation by long PFO was very similar to that by WT PFO, indicating that the AlaAla insertions in each $\beta$-strand didn't significantly alter the pore-forming ability, and that the WT and long PFO make pores with equal efficiency. In contrast, shortening TM segments by two residues greatly reduced pore formation by short PFO. Even after correction for the $\sim 35 \%$ (determined in a previous study (Lin and London 2013)) of short PFO 
that does not bind to model membranes, pore formation by short PFO was reduced about three-fold (after correcting for incomplete short PFO binding to membranes) relative to WT and long PFO, i.e. three to four-fold more short PFO than WT or long PFO was needed to release an equivalent amount of biocytin (Fig. 2).

\section{PFO derivatives with different length TM segments form pores with different diameters}

The difference between short PFO and WT PFO could be explained by either a decrease in pore number, pore size or a combination of both. To investigate if pore size was altered, PFOinduced leakage of macromolecules with different sizes was measured. In the experiment shown in Fig. 3, leakage was measured using vesicles containing entrapped MPTS (MW 538), CB-dextran 3 (MW 3 kD), CB-dextran 10 (MW 10 kD) or CB-dextran 70 (MW 70 kD). The effective diameter of CB-dextran 3 in solution is estimated to be $2.8 \mathrm{~nm}$, that of CB-dextran 10 to be $4.72 \mathrm{~nm}$, and that of CB-dextran 70 to be $11.6 \mathrm{~nm}$ (Scherrer et al. 1971). LUVs containing trapped MPTS or dextrans were exposed to PFO. If pores are sufficiently large, efflux of entrapped molecules should result in their release into the supernatant after pelleting vesicles by centrifugation.

Using these molecules we first examined pore size in LUVs composed of DOPC/BrPC/cholesterol. (BrPC has a high density and 15 mol\% BrPC was used to assist LUV pelleting this lipid mixture, which otherwise does not pellet efficiently.) The amounts of protein used were chosen to give the same amount of membrane-bound WT, short and long PFO based on our previous studies, showing that while long and WT PFO are fully membrane-bound only about $65 \%$ of short PFO binds to membranes (Lin and London 2013), and an easily measured extent of pore formation. As shown in Fig. 3A, the pores formed by both WT and long PFO didn't block the release of MPTS or CB-dextran, with almost $30 \%$ of fluorescence detected in the supernatant. The observation that \% release is independent of dextran size indicates that partial release is not due to slow release of dextran, but rather the fact that most vesicles do not contain any pores. Confirming this, the \% release of both MPTS and CB-dextran 70 by WT and long PFO were almost identical after $1 \mathrm{~h}$ and $2 \mathrm{~h}$ incubation (Supplemental Fig. 2).

Pores formed by short PFO allowed the release of MPTS to a slightly lower extent than WT and long PFO. However, 3kD dextran was released to a much lower extent, and only a minimal leakage of the larger dextrans was observed. This indicates that at least some of the pores formed by short PFO are reduced in size relative to those formed by WT and long PFO. The residual leakage of large dextrans could either mean that there is a range of pore sizes so that a few vesicles had pores large enough to allow a large dextran to escape the vesicle, or that smaller average pore size slows the release of large dextrans. The latter explanation is unlikely because the extent of leakage by $10 \mathrm{kD}$ and $70 \mathrm{kD}$ dextran was similar. Furthermore, for both MPTS and CB-dextran 70, short PFO-induced leakage was similar after $1 \mathrm{~h}$ and $2 \mathrm{~h}$ incubation (Supplemental Fig. 2). Therefore, it appears that most of the pores formed by short PFO are too small to allow molecules of $10 \mathrm{kD}$ or larger through, although a few larger pores form.

PFO pore size was also studied in ternary lipid mixtures (DSPC/DMoPC/cholesterol) forming a mixture of liquid ordered (Lo) and liquid disordered (Ld) domains. In a previous study, we demonstrated that in these lipid vesicles, WT and long PFO prefers the thick Lo domain, whereas short PFO has a higher affinity for thin Ld domain (Lin and London 2013). Vesicles with this composition were used (again under conditions giving complete binding of WT and long 
PFO and maximal binding of short PFO (Lin and London 2013)) to investigate whether different domain localization and/or lipid type would affect PFO pore size. As shown in Fig. 3B, the pattern of leakage was very similar to that observed in DOPC/BrPC/cholesterol vesicles, with pores formed by WT and long PFO allowing an equal extent of leakage ( 30\%) of both MPTS and CB-dextrans, while short PFO pores permit release of MPTS molecules, but not of large CBdextran molecules. This suggests that differences in domain localization and/or lipid type do not greatly influence PFO pore size. The observation that for short PFO release of $10 \mathrm{kD}$ and $70 \mathrm{kD}$ CB-dextrans is close to zero (after subtraction of control) suggests that in the ternary mixture almost all of the pores formed by short PFO are too small to allow molecules of 10kD or larger through.

It should be noted that although we carried out these experiments at low $\mathrm{pH}$, which enhances PFO membrane-insertion, and has been hypothesized to be the physiologically relevant $\mathrm{pH}$, at least under some conditions (O'Brien et al. 2004), we observed similar behavior at $\mathrm{pH} 7$ (Supplemental Fig. 2).

To see if PFO pore size was somehow different for dextrans and proteins, which may not have identical shapes, we examined PFO-induced leakage of BOD-SA trapped in LUVs composed of $\mathrm{DOPC} /$ cholesterol and DSPC/DMoPC/cholesterol. The $\sim 60 \mathrm{kD}$ streptavidin tetramer is globular, with dimensions of $\sim 5.5 \times 4.5 \times 5.0 \mathrm{~nm}$ (Darst et al. 1991). Fig. 4 shows that in both binary and ternary lipid mixtures, the large pores formed by WT or long PFO allowed BOD-SA leakage out of the vesicles. The extent of leakage was similar to that of CB-dextrans. However, very little BOD-SA leakage was observed in the samples containing short PFO. The extent of residual leakage of BOD-SA was very similar to that seen for large dextrans. This further confirms that the pores formed by short PFO are significantly reduced in size.

\section{DISCUSSION}

\section{Properties of Different Forms of PFO}

We previously described the preparation and some properties of PFO molecules in which the segments that form TM $\beta$-strands were either lengthened or shortened. Several properties confirmed that the overall folding of WT, long and short PFO in membranes is very similar (Lin and London 2013). First, all forms of PFO inserted into membranes in a strictly cholesteroldependent fashion, in which the binding and insertion of domain 3 into membranes only occurs above a threshold cholesterol concentration. This indicates that the shortened and lengthened mutants recognize cholesterol, and thus that the cholesterol-binding domain (domain 4) is properly folded. In addition, pore formation had the same strict cholesterol dependence as membrane-insertion (Lin and London 2013). This, and cholesterol-dependent membrane insertion of domain 3 , would not be predicted if pore formation was just due to membrane leakiness resulting from the non-specific interaction of an improperly folded PFO aggregate with membranes.

Lengthened and shortened PFO mutants formed pore-inducing structures with specific properties that can only be explained easily in terms of their assembly into TM $\beta$-barrels. This conclusion arises from the observation that WT PFO forms pores most efficiently in bilayers with a thickness that is typical of a natural bilayer with (lipids with C18 fatty acyl chains) (Lin and London 2013). When membranes are too thick, or too thin, pore formation is less efficient 
(Lin and London 2013). In other words, pore formation is most efficient when there is a nearmatch between the length of the $\beta$-barrel and the width of the bilayer. When TM $\beta$-strands were lengthened or shortened, PFO with lengthened TM segments formed pores maximally in membranes thicker than did WT (bilayers with C20 fatty acyl chain lipids), while PFO with shortened TM segments, formed pores maximally on bilayers thinner than did WT (bilayers with C16 fatty acyl chain lipids). This is exactly what would be expected if these mutations were increasing or decreasing the length of a TM $\beta$-barrel. There is one more line of evidence indicating that the mutated proteins in membranes formed $\beta$-barrels with TM segments that had been lengthened or shortened, rather than non-specific aggregates. When the mutants were incorporated into vesicles with co-existing lipid domains having two different thicknesses, they associated most strongly with the lipid domains having a thickness most closely matching that the length of the TM segments (Lin and London 2013).

Alternate hypotheses for the structure of the TM, pore-forming segments in short PFO are less satisfactory. Since the segments have an alternating hydrophobic-hydrophilic they cannot form an amphiphilic helical structure with a hydrophilic face towards a pore and a hydrophobic face in contact with lipid. Furthermore, since it takes almost twice as many residues to span a bilayer for a helix relative to a $\beta$-strand, it is hard to see how any sort of TM helical hairpin structure could form. Another possibility is that in the case of short PFO the normally pore-forming segments do not form a TM structure at all, and instead pore formation reflects a simple membrane destabilization effect. However, this (or helix formation) does not fit the observed effects of membrane width upon pore formation. In particular, the observation that pores form more efficiently in intermediate width bilayers relative to thin or thick bilayers would not be expected. Instead, membrane destabilization would likely be most severe in the thinnest membranes. This model also does not explain the preference of short PFO to associate with membrane domains having a thin bilayer width.

Thus, although further studies are warranted, we conclude that it is likely that after membrane insertion mutated PFOs form TM $\beta$-barrels, with the barrel lengths expected for the mutations lengthening or shortening the TM forming sequences.

\section{Possible Structural Implications of The Pore-Forming Properties of Mutant and WT PFO}

If we assume that the model in which the changes in TM sequence length result in a shortening or lengthening of the TM $\beta$-barrel is correct, an important question is: what are the implications of pore size for how PFO structure was altered by mutation? In general the size of the pores formed by TM $\beta$-barrel proteins is determined by the number of $\beta$-strands in the barrel and tilt angle, which is a measure of the inclination of the $\beta$-strands relative to the barrel axis (Schulz 2002). The smallest barrels contain $8 \mathrm{TM}$ strands, such as the outer membrane protein A (OmpA) (Pautsch et al. 1998) and the outer membrane phospholipase A (OmpLA) (Snijder et al. 1999). These proteins either do not form pores, or form pores that are very small in diameter (1-2 $\mathrm{nm})$, permitting flux of small molecules but not of fully folded proteins. The largest barrels of known structures are the cholesterol-dependent cytolysins, the class of proteins of which PFO is a member, containing up to 176 strands (Giddings et al. 2003) and creating TM pores of a remarkably large size ( $30 \mathrm{~nm}$ in diameter).

For PFO, a tilt angle of $20^{\circ}$ has been determined by Sato et al. (Sato et al. 2013). Our previous studies showed that in short PFO the two-residue deletion in each TM $\beta$-strand (which 
shortens the strand by 6-7 $\AA$, resulted in 5-6 $\AA$ shortening of $\beta$-barrel TM width (Lin and London 2013), which can be explained by the deletion without a significant change in tilt angle. Furthermore, for the shortened TM-strands to span a lipid bilayer one would predict that, if anything, tilt angle would decrease, which would reduce pore size. This is not what is observed. The small pores formed by short PFO were quite distinct from those formed by WT and long PFO. There appeared to be a size cutoff at close to MW 3000. A more exact size cutoff is difficult to define, because dextran molecules for any given average molecular weight actually have a range of sizes (Sharpe et al. 1999), and could have different degrees of branching, which would also affect their shape and ability to pass through a pore of defined size. In addition, we cannot rule out some of heterogeneity of small pore size due to variations in oligomer size in the 4-5 range. Nevertheless, a size cutoff of $3 \mathrm{kD}$ is very close to what would be predicted for the tetramer suggested by EM. Each PFO monomer normally contributes two membraneinserting $\beta$-hairpins (four $\beta$-strands) to the wall of the pore (Shatursky, Heuck et al. 1999). This means the pore in a tetramer or pentamer would be surrounded by $16-20 \beta$-strands. The $19 \beta$ strand structure of the pore forming protein VDAC has been solved at high resolution (Bayrhuber et al. 2008; Colombini 2012). VDAC forms a pore with a diameter close to $2.5 \mathrm{~nm}$, and size cutoff of $4 \mathrm{kD}$. Since a $16 \beta$-strand barrel should have a cross sectional area only slightly less than that of VDAC (roughly $(16 / 19)^{2}$ that of VDAC if $\beta$-strand number dominates pore size) short PFO should have either a very similar or a slightly smaller size cutoff than VDAC, which is what was observed. Of course, pore size would be affected by the size of the side chains facing the pore interior. Calculation of average side chain volume shows pore-facing VDAC side chains are on the average very slightly larger than those of PFO so that the PFO pore might be slightly larger than predicted by the number of $\beta$-strands (not shown). In addition, formation of a short PFO pore involving a 16-20 $\beta$-strand barrel is also consistent with the 2-nm diameter pores formed by heptametric bacterial toxins aerolysin and staphylococcal $\alpha$-toxin, which are formed by a barrel with $14 \beta$-strands (Wilmsen et al. 1992; Moniatte et al. 1996; Valeva et al. 1997).

It is interesting that the suggestion from EM that short PFO forms a tetramer or a pentamer with a pore fits the pore size data. This is unlikely to be a coincidence, and so the EM results are likely not an artifact. Nevertheless, our conclusions do not depend on the EM data.

Short PFO also allowed a small amount of size-independent release of larger dextran and streptavidin. One likely explanation is that short PFO forms a very small amount of large poreforming oligomers observed for WT and long PFO. Another possibility is that short PFO induces a small amount of vesicle lysis. It should also be noted that we cannot rule out the possibility that short PFO pore size, or the ratio of small to large pores formed by short PFO, would be dependent on PFO concentration.

Although a decrease in oligomer size explains the small pore size observed with short PFO, it does not explain why shortening TM segments alters oligomer size. Presumably, the deletion of $\beta$-strand residues alters inter-strand interaction between neighboring subunits. It has been found that monomer-monomer association involves hydrogen bonding of the $\beta 1$ strand from one PFO molecule to the $\beta 4$ strand of the adjacent molecule (Ramachandran et al. 2004). The two residue deletion in short PFO may alter the monomer-monomer interfacial surface, so as to weaken subunit interaction and/or block PFO oligomer growth, alter inter-strand contacts, or alter $\beta$-barrel curvature in such a fashion as to result in smaller oligomers or pores. 
It should also be noted that the change in structure might not be strictly due to the shortening of the TM segments, but rather due to altered interactions that result from the specific residues chosen for deletion. It is entirely possible that a different form of shorted PFO, in which different residues in the TM sequences were deleted, would form a normal oligomer and have a wild-type pore size.

Formation of a structure with a small pore by short PFO is somewhat surprising based on the conventional process by which PFO pores are assembled. It is thought that a large PFO oligomer assembles into a prepore prior to the interaction of the helical loops that form the $\beta$ barrel pore with the lipid bilayer. If the radius of the PFO ring were irreversibly determined at the prepore assembly step, and if the helical loops play no role in the oligomerization, the short mutant should have formed a large pore. However, it has not been shown that $100 \%$ of PFO always forms the "classical" large oligomer prepore. A few percent of WT might form a small oligomer. A minor component might have been overlooked. In the short mutant, the equilibrium could favor assembly into a smaller prepore oligomer. Second, it has recently been found that the conformations formed by membrane-inserted PFO can be reversible (Lin et al. 2014). A prepore might re-equilibrate after assembly between smaller and larger oligomer states, again with the large form predominating except for short PFO.

Long PFO formed the large pores characteristic of cholesterol-dependent cytolysins. The extent of membrane binding (Lin and London 2013), oligomer size, as estimated from EM, pore formation and pore size were quite similar to that of WT PFO. The observation that long and WT PFO have similar pore formation and pore size suggests that the lengthened TM segments either have a greater tendency to adjust TM length by increased tilting, or the Ala insertions at the end of $\beta$-hairpin loops do not alter inter-strand interactions.

The release of entrapped molecules by WT, short and long PFO was incomplete under the conditions we studied. This indicates that only a subpopulation of vesicles had pores. However, we estimated that there were enough PFO molecules to form 2-3 pores per vesicles, if all PFO is in the pore-forming state. Thus, either some PFO was not in the pore-forming conformation, or pores were non-randomly distributed so that some vesicles had an insufficient number of PFO molecules to form pores. The latter behavior could be present if PFO undergoes cooperative insertion into membranes, in which binding of one PFO to a vesicle help recruit other PFO molecules.

It should be noted that shortening or lengthening PFO TM segments affects some properties of PFO in addition to pore formation. First, altering TM length alters the strength of oligomerization. Neither long nor short PFO forms the SDS-resistant oligomers observed with WT PFO (Shepard, Shatursky et al. 2000). Second, as shown previously, the mutated segments have somewhat different local conformations in the segment in aqueous solution. The sequences forming the TM $\beta$-hairpins after membrane insertion are packed as two sets of three $\alpha$-helices when in aqueous solution prior to membrane insertion (Shepard, Heuck et al. 1998; Shatursky, Heuck et al. 1999). Acrylodan labeling studies show that the environment of residue 215 in these hairpins is more hydrophobic for long and short PFO with (acrylodan emission $\lambda_{\max }$ $=483 \mathrm{~nm}$ ), than for WT PFO $\left(\lambda_{\max }=504 \mathrm{~nm}\right.$ ) (Lin and London 2013). Finally, proper folding of short PFO was less (66\%) than that of WT and long PFO based on the fraction of molecules that bind to lipid (Lin and London 2013). 


\section{Biological and biomedical applications of pore-forming proteins with different pore and transmembrane lengths.}

Relative to multi-TM $\alpha$-helix proteins, $\beta$-barrel structures are especially amenable to engineering of TM segment properties to adjust their properties for various applications. Prior studies have demonstrated that various modifications in the coding genes can be introduced without affecting $\beta$-barrel structure in membranes (Koebnik 1999). This, added to the range of pore sizes that can be formed by such proteins (see above), makes them attractive targets for engineering pores with various sizes.

Many mutations in pore-forming $\beta$-barrel proteins have the goal of altering pore properties. Widely used approaches for pore-forming protein engineering mainly involve straight genetic engineering or targeted chemical modification (Bayley et al. 2004). Several mutations in the pore eyelet of the porin from Rhodopseudomonas blastica, have been reported (Schmid et al. 1998), altering size and charge selectivity of the pore. In another study, a substitution introducing either 7 or 14 Arg near the narrowest part of the pore formed by the heptameric $\alpha$-hemolysin protein acted as primitive binding site for channel blockers (Cheley et al. 2002). Other studies have shown modification of residues on the interior of the $\alpha$-hemolysin cap with a covalently bound poly(ethylene glycol) (PEG) chain (Movileanu et al. 2000) or introducing a non-covalent adaptor $\beta$-cyclodextrin within its lumen modulate the size and selectivity of $\alpha$-hemolysin pores (Yannakopoulou et al. 2011). Our results provide an additional new approach for engineering the properties of pore-forming proteins. By altering the TM $\beta$ strand lengths we have shown the architectural arrangement of $\beta$-strands can be changed in a fashion that alters $\beta$-barrel and pore size. It is even possible that hybrid oligomers with a wider range of sizes could be generated by mixing mutant PFO and WT PFO with different ratios.

Pore-forming proteins can be used for permeabilization of cells (Walev et al. 2001), and engineering pore size could lead to useful applications in medicine to attack malignant cells or for drug delivery. There may be additional applications for $\beta$-barrel proteins with engineered TM lengths. As noted above, we recently demonstrated that due to hydrophobic mismatch between TM barrel length and membrane domains width, the length of TM segments affects multi-TM segment protein partitioning into lipid rafts (Lin and London 2013). This indicates that engineered PFO pores could be useful for local targeting. For example, through coupling to engineered PFO, drugs or other macromolecules could be introduced so as to interact with specific proteins located within or outside of raft domains in cell membranes.

Acknowledgement - The authors thank Prof. A. Heuck, (U. Mass. Amherst) for providing protocols to prepare BODIPY-labeled streptavidin. 


\section{REFERENCES}

Aroian R and Van Der Goot FG (2007). Pore-forming toxins and cellular non-immune defenses (CNIDs). Current Opinion in Microbiology 10: 57-61.

Bayley $\mathrm{H}$ and Jayasinghe $\mathrm{L}$ (2004). Functional engineered channels and pores (Review). Mol Membr Biol 21: 209-220.

Bayrhuber M, Meins T, Habeck M, Becker S, Giller K, Villinger S, Vonrhein C, Griesinger C, Zweckstetter $\mathrm{M}$ and Zeth $\mathrm{K}$ (2008). Structure of the human voltage-dependent anion channel. Proc Natl Acad Sci U S A 105: 15370-15375.

Bhakdi S and Tranum-Jensen J (1984). Mechanism of complement cytolysis and the concept of channel-forming proteins. Philos Trans R Soc Lond B Biol Sci 306: 311-324.

Cassidy SK and O'riordan MX (2013). More than a pore: the cellular response to cholesteroldependent cytolysins. Toxins (Basel) 5: 618-636.

Cheley S, Gu LQ and Bayley H (2002). Stochastic sensing of nanomolar inositol 1,4,5trisphosphate with an engineered pore. Chem Biol 9: 829-838.

Cheng HT and London E (2011). Preparation and properties of asymmetric large unilamellar vesicles: interleaflet coupling in asymmetric vesicles is dependent on temperature but not curvature. Biophys J 100: 2671-2678.

Chiu J, March PE, Lee R and Tillett D (2004). Site-directed, Ligase-Independent Mutagenesis (SLIM): a single-tube methodology approaching 100\% efficiency in $4 \mathrm{~h}$. Nucleic Acids Res 32: e174.

Chiu J, Tillett D, Dawes IW and March PE (2008). Site-directed, Ligase-Independent Mutagenesis (SLIM) for highly efficient mutagenesis of plasmids greater than 8kb. J Microbiol Methods 73: 195-198.

Colombini M (2012). VDAC structure, selectivity, and dynamics. Biochim Biophys Acta 1818: 1457-1465.

Darst SA, Ahlers M, Meller PH, Kubalek EW, Blankenburg R, Ribi HO, Ringsdorf H and Kornberg RD (1991). Two-dimensional crystals of streptavidin on biotinylated lipid layers and their interactions with biotinylated macromolecules. Biophys J 59: 387-396.

Fussle R, Bhakdi S, Sziegoleit A, Tranum-Jensen J, Kranz T and Wellensiek HJ (1981). On the mechanism of membrane damage by Staphylococcus aureus alpha-toxin. Journal of Cell Biology 91: 83-94.

Giddings KS, Johnson AE and Tweten RK (2003). Redefining cholesterol's role in the mechanism of the cholesterol-dependent cytolysins. Proc Natl Acad Sci U S A 100: 11315-11320. 
Hotze EM and Tweten RK (2012). Membrane assembly of the cholesterol-dependent cytolysin pore complex. Biochim Biophys Acta 1818: 1028-1038.

Koebnik R (1999). Structural and functional roles of the surface-exposed loops of the betabarrel membrane protein OmpA from Escherichia coli. J Bacteriol 181: 3688-3694.

Lin $Q$ and London E (2013). Altering hydrophobic sequence lengths shows that hydrophobic mismatch controls affinity for ordered lipid domains (rafts) in the multitransmembrane strand protein perfringolysin O. J Biol Chem 288: 1340-1352.

Lin $Q$ and London $E$ (2014). The influence of natural lipid asymmetry upon the conformation of a membrane-inserted protein (perfringolysin O). J Biol Chem 289: 5467-5478.

Ludtke SJ, Baldwin PR and Chiu W (1999). EMAN: semiautomated software for high-resolution single-particle reconstructions. J Struct Biol 128: 82-97.

Mitsui K, Sekiya T, Okamura S, Nozawa Y and Hase J (1979). Ring formation of perfringolysin $O$ as revealed by negative stain electron microscopy. Biochim Biophys Acta 558: 307-313.

Moniatte M, Van Der Goot FG, Buckley JT, Pattus F and Van Dorsselaer A (1996).

Characterisation of the heptameric pore-forming complex of the Aeromonas toxin aerolysin using MALDI-TOF mass spectrometry. FEBS Lett 384: 269-272.

Movileanu L, Howorka S, Braha O and Bayley H (2000). Detecting protein analytes that modulate transmembrane movement of a polymer chain within a single protein pore. Nat Biotechnol 18: 1091-1095.

Nelson LD, Chiantia S and London E (2010). Perfringolysin O association with ordered lipid domains: implications for transmembrane protein raft affinity. Biophys J 99: 3255-3263.

Nelson LD, Johnson AE and London E (2008). How interaction of perfringolysin O with membranes is controlled by sterol structure, lipid structure, and physiological low pH - Insights into the origin of perfringolysin O-lipid raft interaction. Journal of Biological Chemistry 283: 4632-4642.

O'brien DK and Melville SB (2004). Effects of Clostridium perfringens alpha-toxin (PLC) and perfringolysin $\mathrm{O}$ (PFO) on cytotoxicity to macrophages, on escape from the phagosomes of macrophages, and on persistence of C. perfringens in host tissues. Infect Immun 72: 5204-5215.

Parker MW and Feil SC (2005). Pore-forming protein toxins: from structure to function. Progress in Biophysics \& Molecular Biology 88: 91-142.

Pautsch A and Schulz GE (1998). Structure of the outer membrane protein A transmembrane domain. Nat Struct Biol 5: 1013-1017. 
Ramachandran R, Tweten RK and Johnson AE (2004). Membrane-dependent conformational changes initiate cholesterol-dependent cytolysin oligomerization and intersubunit beta-strand alignment. Nature Structural \& Molecular Biology 11: 697-705.

Rosconi MP, Zhao G and London E (2004). Analyzing topography of membrane-inserted diphtheria toxin T domain using BODIPY-streptavidin: at low $\mathrm{pH}$, helices 8 and 9 form a transmembrane hairpin but helices 5-7 form stable nonclassical inserted segments on the cis side of the bilayer. Biochemistry 43: 9127-9139.

Rossjohn J, Feil SC, Mckinstry WJ, Tweten RK and Parker MW (1997). Structure of a cholesterolbinding, thiol-activated cytolysin and a model of its membrane form. Cell 89: 685-692.

Sato TK, Tweten RK and Johnson AE (2013). Disulfide-bond scanning reveals assembly state and beta-strand tilt angle of the PFO beta-barrel. Nat Chem Biol 9: 383-389.

Scherrer R and Gerhardt P (1971). Molecular sieving by the Bacillus megaterium cell wall and protoplast. J Bacteriol 107: 718-735.

Schmid B, Maveyraud L, Kromer M and Schulz GE (1998). Porin mutants with new channel properties. Protein Sci 7: 1603-1611.

Schulz GE (2002). The structure of bacterial outer membrane proteins. Biochim Biophys Acta 1565: 308-317.

Sharpe JC and London E (1999). Diphtheria toxin forms pores of different sizes depending on its concentration in membranes: probable relationship to oligomerization. J Membr Biol 171: 209221.

Shatursky O, Heuck AP, Shepard LA, Rossjohn J, Parker MW, Johnson AE and Tweten RK (1999). The mechanism of membrane insertion for a cholesterol-dependent cytolysin: a novel paradigm for pore-forming toxins. Cell 99: 293-299.

Shepard LA, Heuck AP, Hamman BD, Rossjohn J, Parker MW, Ryan KR, Johnson AE and Tweten RK (1998). Identification of a membrane-spanning domain of the thiol-activated pore-forming toxin Clostridium perfringens perfringolysin O: an alpha-helical to beta-sheet transition identified by fluorescence spectroscopy. Biochemistry 37: 14563-14574.

Shepard LA, Shatursky O, Johnson AE and Tweten RK (2000). The mechanism of pore assembly for a cholesterol-dependent cytolysin: formation of a large prepore complex precedes the insertion of the transmembrane beta-hairpins. Biochemistry 39: 10284-10293.

Snijder HJ, Ubarretxena-Belandia I, Blaauw M, Kalk KH, Verheij HM, Egmond MR, Dekker N and Dijkstra BW (1999). Structural evidence for dimerization-regulated activation of an integral membrane phospholipase. Nature 401: 717-721. 
Tang G, Peng L, Baldwin PR, Mann DS, Jiang W, Rees I and Ludtke SJ (2007). EMAN2: an extensible image processing suite for electron microscopy. J Struct Biol 157: 38-46.

Tweten RK (2005). Cholesterol-dependent cytolysins, a family of versatile pore-forming toxins. Infection and Immunity 73: 6199-6209.

Valeva A, Palmer M and Bhakdi S (1997). Staphylococcal alpha-toxin: formation of the heptameric pore is partially cooperative and proceeds through multiple intermediate stages. Biochemistry 36: 13298-13304.

Walev I, Bhakdi SC, Hofmann F, Djonder N, Valeva A, Aktories K and Bhakdi S (2001). Delivery of proteins into living cells by reversible membrane permeabilization with streptolysin-O. Proc Natl Acad Sci U S A 98: 3185-3190.

Wang J and London E (2009). The membrane topography of the diphtheria toxin T domain linked to the a chain reveals a transient transmembrane hairpin and potential translocation mechanisms. Biochemistry 48: 10446-10456.

Wilmsen HU, Leonard KR, Tichelaar W, Buckley JT and Pattus F (1992). The aerolysin membrane channel is formed by heptamerization of the monomer. EMBO J 11: 2457-2463.

Yannakopoulou K, Jicsinszky L, Aggelidou C, Mourtzis N, Robinson TM, Yohannes A, Nestorovich EM, Bezrukov SM and Karginov VA (2011). Symmetry requirements for effective blocking of pore-forming toxins: comparative study with alpha-, beta-, and gamma-cyclodextrin derivatives. Antimicrob Agents Chemother 55: 3594-3597. 


\section{FIGURE LEGENDS}
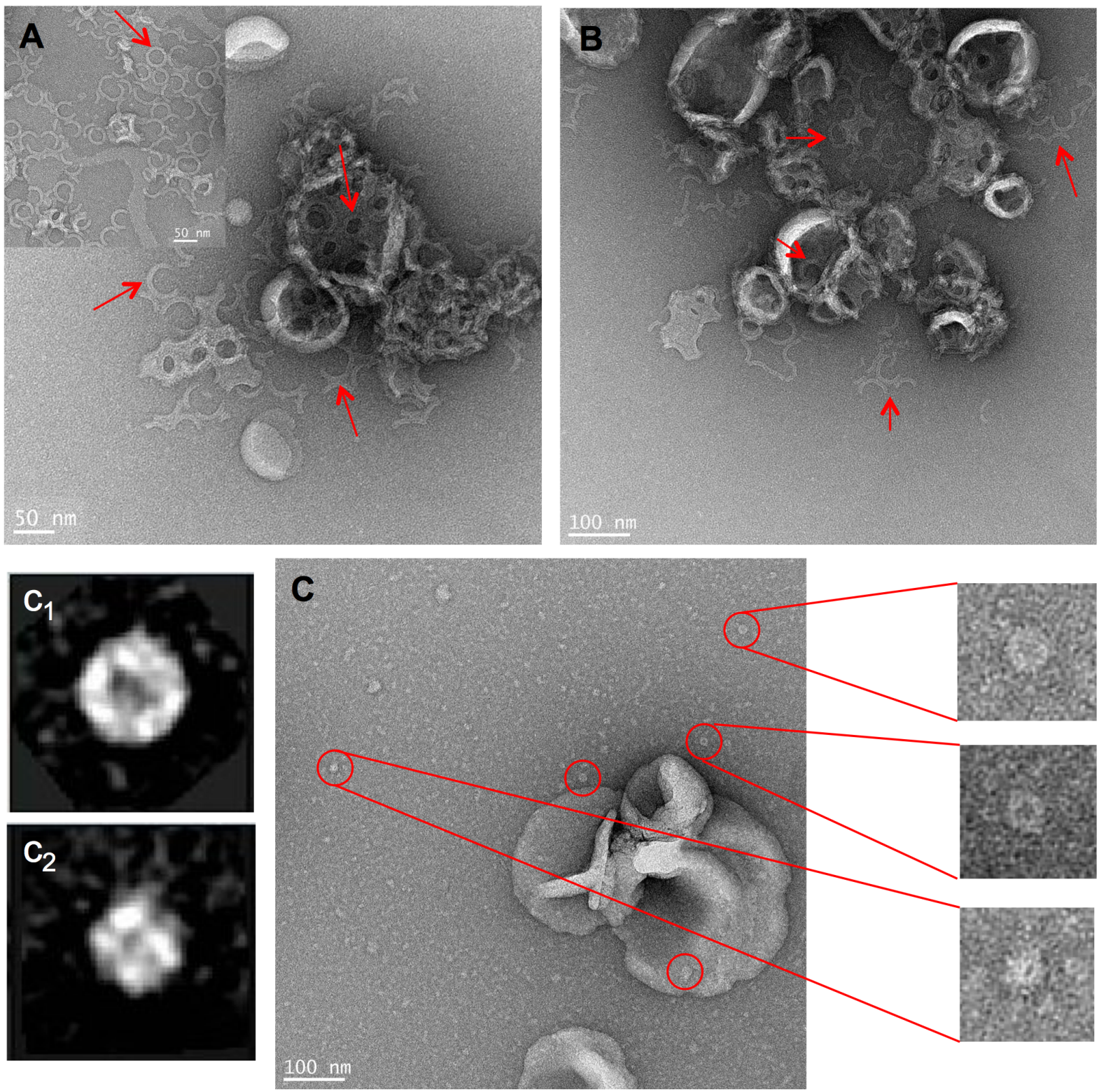

Fig 1. EM image of PFO mutants. (A) $50 \mu \mathrm{g}$ WT PFO in 27.5:27.5:45 (mol:mol:mol) DSPC/DMoPC/cholesterol (500 $\mu \mathrm{M}$ total lipid). Insert in panel A: $20 \mu \mathrm{g}$ WT PFO in 6:4 (mol:mol) DOPC/cholesterol (200 $\mu \mathrm{M}$ total lipid); (B) $50 \mu \mathrm{g}$ long PFO in 27.5:27.5:45 (mol:mol:mol) DSPC/DMoPC/cholesterol (500 $\mu \mathrm{M}$ total lipid). (C) $50 \mu \mathrm{g}$ short PFO in 27.5:27.5:45 (mol:mol:mol) DSPC/DMoPC/cholesterol (500 $\mu \mathrm{M}$ total lipid). Samples were prepared in PBS buffer $\mathrm{pH}$ 5.1. Large objects in each micrograph are lipid vesicles. Arrows in A. and B. point to full or partial PFO rings, many of which have fallen off the vesicles. Circles in C. point to small oligomers, several of which are shown magnified. Averaged 2D views of those small pore particles presented in $C$. are shown: $\left(C_{1}\right)$ an averaged 2D view computed from 17 small pore particles; $\left(C_{2}\right)$ an averaged $2 \mathrm{D}$ view computed from 27 small pore particles. The outer diameter of the averaged particle is $\sim 11-15 \mathrm{~nm}$. The interior lowest density region is $\sim 2-3 \mathrm{~nm}$ in size. 


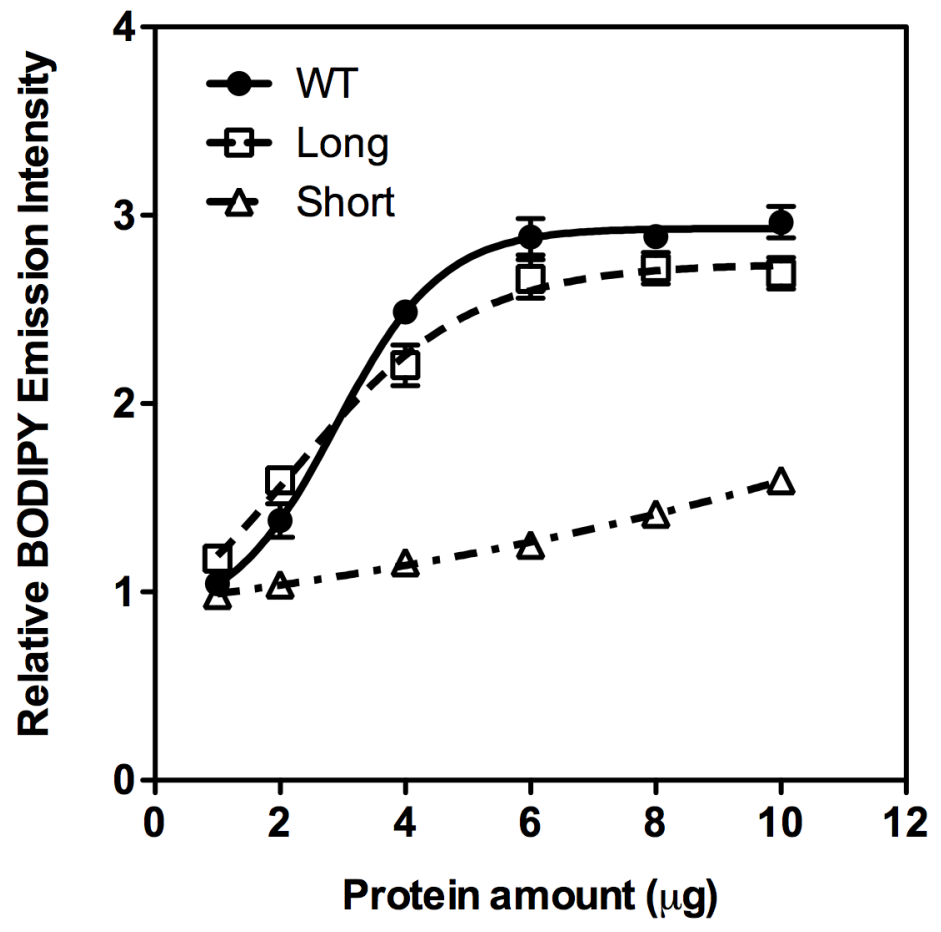

Fig 2. Dose dependent of PFO pore formation. The yaxis shows the increase of external BODIPY-streptavidin fluorescence after the addition of PFO relative to that before the PFO addition. Samples contained LUV composed of $6: 4 \mathrm{~mol} / \mathrm{mol}$ DOPC/cholesterol $(100 \mu \mathrm{M}$ total lipid) containing entrapped biocytin and $10 \mathrm{nM}$ externally added BODIPY-streptavidin in PBS pH 5.1. BODIPY fluorescence was measured $40 \mathrm{~min}$ after the addition of PFO. Average (mean) values and S.D. values (error bars) were obtained from triplicates. 
A

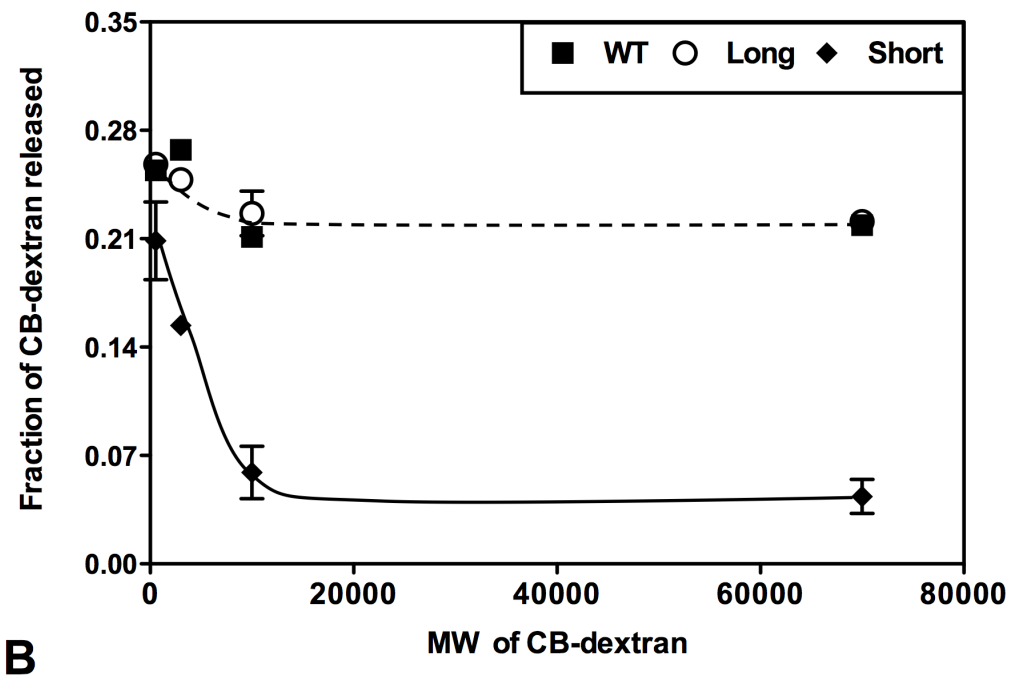

B

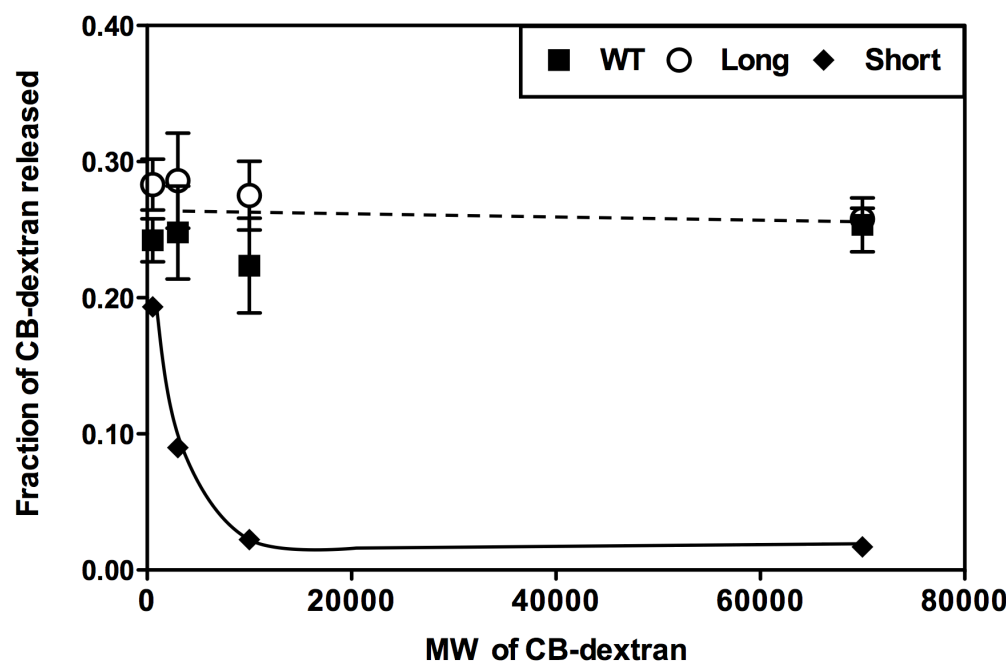

Fig 3. Pore size of PFO mutants detected by PFO-induced CB-dextran leakage. (A) LUVs composed of 45:15:40 (mol:mol:mol) DOPC/BrPC/cholesterol with 0.002 mol\% Rho-DOPE; (B) LUVs composed of $27.5: 27.5: 45 \mathrm{~mol} / \mathrm{mol} / \mathrm{mol}$ DSPC/DMoPC/cholesterol with $0.002 \mathrm{~mol} \%$ RhoDOPE. Samples containing LUVs ( $500 \mu \mathrm{M}$ lipid) entrapped MPTS or CB-dextran without (control) or with $20 \mu \mathrm{g}$ WT (filled square), $20 \mu \mathrm{g}$ long (open circle) or $30 \mu \mathrm{g}$ short (filled diamond) PFO were incubated for $1 \mathrm{~h}$. Final $\mathrm{pH}$ 5.3-5.7. After centrifugation, Rho-DOPE ( $\left.F_{\text {Rho }}\right)$ and MPTS/cascade blue $\left(F_{C B}\right)$ emission intensity were measured for the resuspended pellet and supernatant. The fraction of MPTS (Cascade Blue dye) or CB-dextran released, correcting for incomplete pelleting of lipid, as assayed by the amount of Rho-DOPE in the supernatant, is equal to $\left(\left[\mathrm{F}_{\mathrm{CB} \text {-supernatant }} /\left(\mathrm{F}_{\mathrm{CB} \text {-supernatant }}+\mathrm{F}_{\mathrm{CB} \text {-pellet }}\right)\right]-\left[\mathrm{F}_{\text {Rho-supernatant }} /\left(\mathrm{F}_{\text {Rho-supernatant }}+\mathrm{F}_{\text {Rho-pellet }}\right)\right]\right) /\left[1-\left[\mathrm{F}_{\text {Rho- }}\right.\right.$ supernatant $\left./\left(F_{\text {Rho-supernatant }}+F_{\text {Rho-pellet }}\right)\right]$. Values shown after subtraction of the background which is due to the amount of MPTS or CB-dextran unassociated with vesicles in the absence of PFO (The background fraction in the supernatant was $\leq 0.05$ ). Average (mean) values and S.D. values (error bars) were obtained from triplicates. 
A

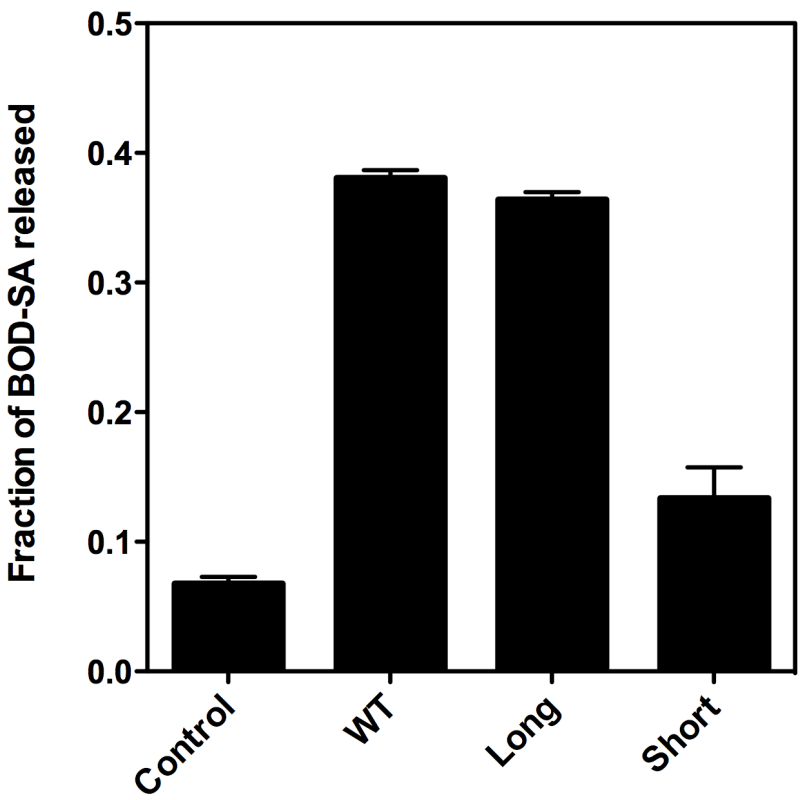

B

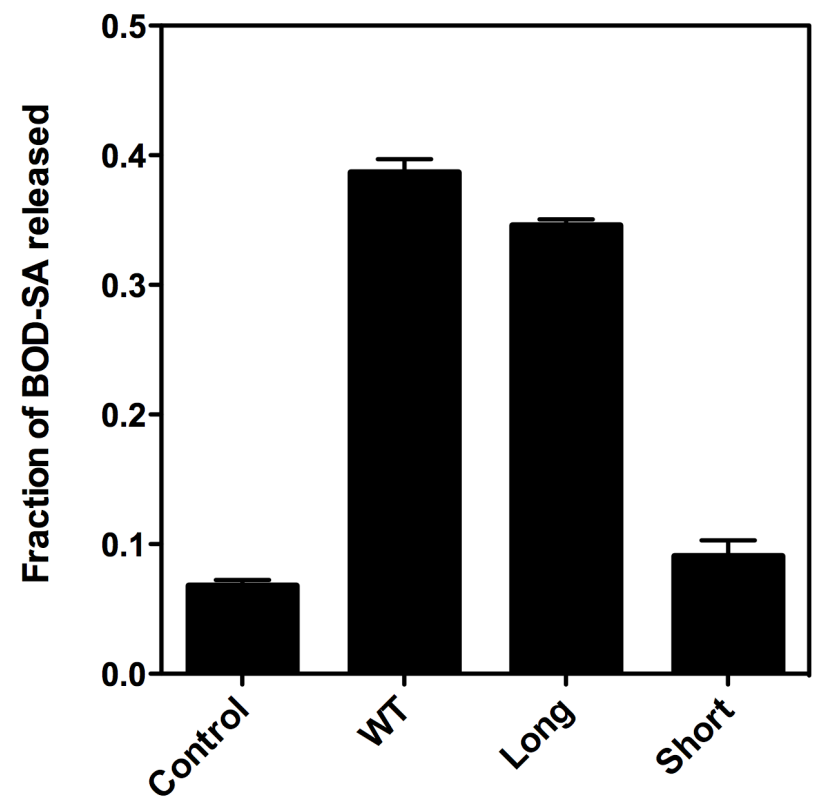

Fig 4. Pore size of PFO mutants detected by PFO-induced BOD-SA leakage. (A) LUVs composed of 45:15:40 (mol:mol:mol) DOPC/BrPC/cholesterol with 0.002 mol\% Rho-DOPE; (B) LUVs composed of 27.5:27.5:45 mol/mol/mol DSPC/DMoPC/cholesterol with 0.002 mol\% Rho-DOPE. Samples containing LUVs ( $500 \mu \mathrm{M}$ lipid) entrapped BOD-SA without (Control) or with $20 \mu \mathrm{g}$ $\mathrm{WT}, 20 \mu \mathrm{g}$ long or $30 \mu \mathrm{g}$ short PFO were incubated for $1 \mathrm{~h}$. Final pH 5.3-5.7. After centrifugation rhodamine and BODIPY emission intensity were both measured for the resuspended pellet and supernatant. Fraction of BOD-SA in the supernatant was calculated similarly to the method in Figure 3. Average (mean) values and S.D. values (error bars) were obtained from triplicates. 